\title{
HPV prevalence and genotypes in different histological subtypes of cervical adenocarcinoma, a worldwide analysis of 760 cases
}

Edyta C Pirog ${ }^{1}$, Belen Lloveras ${ }^{2}$, Anco Molijn ${ }^{3}$, Sara Tous ${ }^{4}$, Núria Guimerà ${ }^{3}$, Maria Alejo ${ }^{5}$, Omar Clavero ${ }^{4}$, Joellen Klaustermeier ${ }^{4,6}$, David Jenkins ${ }^{3}$, Wim GV Quint ${ }^{3}$, Francesc Xavier Bosch ${ }^{4}$, Laia Alemany ${ }^{4,6,7}$, Silvia de Sanjosée,6,7 and on behalf of the RIS HPV TT study group

${ }^{1}$ Department of Pathology, Weill Medical College of Cornell University, New York, NY, USA; ${ }^{2}$ Department of Pathology, Hospital del Mar, Barcelona, Spain; ${ }^{3}$ DDL Diagnostic Laboratory, Rijswijk, The Netherlands; ${ }^{4}$ Unit of Infections and Cancer, Cancer Epidemiology Research Programme, Institut Català d'Oncologia, Barcelona, Spain; ${ }^{5}$ Department of Pathology, Hospital General de L'Hospitalet, Barcelona, Spain and ${ }^{6}$ CIBER en Epidemiología y Salud Pública (CIBERESP), Barcelona, Spain

The goal of our study was to provide comprehensive data on the worldwide human papillomavirus (HPV) genotype distribution in patients with invasive cervical adenocarcinoma in correlation with histologic tumor subtypes, geographical location, patients' age, and duration of sample storage. Paraffin-embedded samples of 760 cervical adenocarcinoma cases were collected worldwide. A three-level pathology review of cases was performed to obtain consensus histologic diagnoses and 682 cases were determined to be eligible for further analysis. HPV DNA detection and genotyping was performed using SPF-10/DEIA/LiPA 25 system (version 1). Classic cervical adenocarcinoma accounted for $83.1 \%$ of cases, while rare histological variants accounted for a few percent of cases individually. HPV positivity varied significantly between the different histologic tumor subtypes. Classic cervical adenocarcinoma showed high HPV positivity $(71.8 \%)$, while other adenocarcinoma types had significantly lower HPV prevalence (endometrioid $27.3 \%$, serous $25 \%$, clear cell $20 \%$, not otherwise specified $13.9 \%$, and minimal deviation $8.3 \%$ ). In all, $91.8 \%$ of HPV-positive tumors showed the presence of a single viral type and in $7 \%$ of cases multiple viral types were detected. Three HPV genotypes, HPV 16, 18, and 45 , dominated in all adenocarcinomas and together accounted for $94.1 \%$ of HPV-positive tumors. HPV16 was the most common and found in 50.9\% of HPV-positive cases, followed by HPV18 (31.6\%) and HPV45 (11.6\%). HPV prevalence varied depending on geographical region, patient age, and sample storage time. Tumors from older patients and tumor samples with longer storage time showed lower HPV prevalence. Our results indicate that HPV vaccines may prevent up to $82.5 \%$ (HPV16/18) and up to $95.3 \%$ (9-valent vaccine) of HPV-positive cervical adenocarcinomas, mostly the classic type. HPV testing and vaccination will not provide full coverage for a very small subset of classical adenocarcinomas and most of the rare tumor variants such as clear cell, serous, endometrioid, and minimal deviation.

Modern Pathology (2014) 27, 1559-1567; doi:10.1038/modpathol.2014.55; published online 25 April 2014

Keywords: cervical adenocarcinoma; human papillomavirus

Correspondence: Dr S de Sanjosé, MD, PhD, Unit of Infections and Cancer, Cancer Epidemiology Research Programme, Institut Català d'Oncologia-Catalan Institute of Oncology, Gran Via de l'Hospitalet, 199-203, L’Hospitalet de Llobregat, Barcelona 08908, Spain.

E-mail: s.sanjose@iconcologia.net

${ }^{7}$ Equally contributed as senior authors.

Received 28 December 2013; accepted 28 January 2014; published online 25 April 2014
With the introduction of human papillomavirus (HPV) vaccines the prevention of cervical cancer entered a new stage. Currently approved vaccines targeting two oncogenic HPV types, HPV 16 and HPV 18, are hoped to prevent at least $70 \%$ cases of cervical cancer. Besides cross-protection over the existing vaccines the work on new vaccines is on-going with the goal to expand vaccine coverage 
to other viral types. With this aim in sight, the information about individual HPV genotypes responsible for malignant transformation of cervical mucosa has pertinent, practical significance. Our recent study of 10575 cases of invasive cervical cancer carried out at the Catalan Institute of Oncology ${ }^{1}$ was the largest assessment of HPV genotypes to date. HPV typing of cancer cases has identified most common genotypes as HPV16, 18, $31,33,35,45,52$, and 58 , and it has been recommended that these types should be given priority when the cross-protective effects of current vaccines are assessed, and that they should be considered in the second generation of polyvalent HPV vaccines. The same study revealed that in contrast to squamous cell carcinomas in which HPV was detected in $87 \%$ of cases, only $62 \%$ of adenocarcinomas were found to be HPV positive. Given this high rate of HPV negativity in adenocarcinomas we decided to conduct a substudy to elucidate the potential reasons for this finding. One of the possible explanations was that HPV negativity could be due to misclassification of cases submitted by contributing institutions, for example, inclusion of endometrial cancers with secondary cervical involvement or tumors metastatic to the cervix. In addition, some of the histological subtypes of cervical adenocarcinomas have been previously reported as not related to HPV infection. The relative contribution of such cases was unknown since detailed histopathologic review was not performed.

To get better understanding of the role of HPV infection in pathogenesis of cervical adenocarcinoma, we performed a three-level pathology review of all adenocarcinoma cases to obtain consensus histologic diagnoses. We then correlated the results of HPV detection with the consensus histologic diagnoses, patients' age, duration of sample storage, and geographical location.

\section{Materials and methods}

\section{Case Accrual}

A retrospective cross-sectional study was designed and coordinated by the Institut Català d'Oncologia, (ICO) Barcelona, Spain, and DDL Diagnostic Laboratory (DDL), Rijswijk, The Netherlands, to evaluate the distribution of HPV types in women with invasive cervical cancer diagnosed from 1940 to $2009 .{ }^{1}$

The specimens for the overall cervical cancer series were obtained from hospital pathology archives in 38 countries-Europe (Bosnia-Herzegovina, Croatia, Czech Republic, France, Greece, Italy, The Netherlands, Poland, Portugal, and Spain); North America (USA); Latin America (Argentina, Brazil, Chile, Colombia, Guatemala, Honduras, Mexico, Paraguay, Peru, and Venezuela); Africa (Algeria, Mozambique, Nigeria, and Uganda);
Asia (Bangladesh, China, India, Israel, Japan, South Korea, Kuwait, Lebanon, Philippines, Taiwan, Thailand, and Turkey); and Oceania (Australia).

From the global study including 10575 invasive cervical cancer cases, 760 paraffin-embedded tumor samples from consecutive cases of cervical adenocarcinoma were obtained. Additionally, information about age at diagnosis, year of diagnosis, and original histological diagnosis was received from the participating centers. For each case, we received one most representative paraffin-embedded tissue block from the tumor. Paraffin blocks were processed under strict conditions to avoid contamination. Four paraffin sections were systematically obtained from each block using a sandwich method. First and fourth sections were used for histopathological assessment after hematoxylin and eosin staining, and the second and third sections were used for HPV DNA detection and genotyping. All protocols were approved by the local and ICO ethics committees, and the entire study progress was overseen by an International Steering Committee.

\section{Histopathologic Review of Adenocarcinomas}

All pathology evaluations were performed based on the hematoxylin-eosin stained slides obtained from the paraffin blocks. The cases were subclassified according to a simplified scheme of WHO adenocarcinoma classification (Table 1). ${ }^{2}$ This simplified classification was developed as a tool for enhanced reproducibility of pathologic diagnosis in multicenter studies of cervical adenocarcinoma. ${ }^{3}$ All adenocarcinoma cases were first reviewed by three ICO pathologists: OC, BLL, and MA and then independently by DDL-associated pathologist, ECP. The reviews were blinded between the institutions and blinded to the results of HPV testing. Cases with discrepant diagnoses were re-assessed and re-classified in a consensus review by BLL and ECP. In this multistep histopathologic review, the final diagnoses for the cases were established.

\section{HPV Detection and Typing}

Cases were analyzed at the ICO and DDL HPV laboratories for the presence of HPV DNA. HPV DNA detection was done using PCR with SPF-10 broad-spectrum primers followed by DNA enzyme immunoassay. Genotyping was performed with a reverse hybridization line probe assay, $\mathrm{LiPA}_{25}$ (version 1, Laboratory Biomedical Products, Rijswijk, The Netherlands). $\mathrm{LiPA}_{25}$ enables simultaneous detection of individual 25 high-risk and lowrisk HPV types $(6,11,16,18,31,33,34,35,39,40$, $42,43,44,45,51,52,53,54,56,58,59,66,68,70$, and 74$)$. The exact assay conditions were described previously. ${ }^{1}$ 
Table 1 Simplified classification of cervical adenocarcinomas with corresponding grouping of WHO tumor categories and previously reported rate of HPV positivity

\begin{tabular}{lll} 
Classification & Adenocarcinoma subtypes as per WHO classification & $\begin{array}{l}\text { Reported rate of HPV } \\
\text { positivity }\end{array}$ \\
\hline Classic & $\begin{array}{l}\text { Classic adenocarcinoma of endocervix including usual endocervical type, } \\
\text { villoglandular type, intestinal type }\end{array}$ & High rate of HPV positivity \\
Serous & Serous adenocarcinoma of cervix & Low rate of HPV positivity \\
Clear cell & Clear-cell adenocarcinoma and mesonephric adenocarcinoma of cervix & Low rate of HPV positivity \\
Minimal deviation & Minimal deviation adenocarcinoma including gastric type & Low rate or no HPV positivity \\
Endometrioid & Endometrioid adenocarcinoma of upper endocervix & Variable HPV positivity \\
Not otherwise & All cervical adenocarcinoma samples that cannot be categorized under other & Variable HPV positivity \\
specified & diagnostic codes & \\
\hline
\end{tabular}

\section{Statistical Analysis}

HPV DNA proportions and 95\% confidence intervals were determined for the different histologies, geographical regions, age at diagnosis, and year of diagnosis. Unconditional logistic regression analysis was used to evaluate HPV negativity among classic cervical adenocarcinomas for a given variable, taking into account other relevant variables, and squamous cell carcinomas as a comparison group (from the same study). The best fitting model was selected based on the log-likelihood ratio test. We have selected only the classic cervical adenocarcinomas because it is the most clear HPVassociated cervical adenocarcinoma subtype and to eliminate any noise in the modeling process and results derived from including other less frequent and probably not HPV-related histological subtypes.

HPV type distribution was estimated among HPV DNA-positive cases and adding multiple infections to single types by using a weighting algorithm previously described. ${ }^{1}$

Statistical significance for all analysis was set at the two-sided 0.05 level. Data analyses were performed with the Statistical Package for the Social Sciences (SPSS) version 13.0 (SPSS Inc., Chicago, IL, USA) and with STATA version 10.0 (Stata Corporation, Computing Resource Center, College Station, TX, USA).

\section{Results}

A total of 760 cases of cervical adenocarcinoma were obtained from the participating institutions. ${ }^{1}$ Following the multistep consensus histologic review 682 cases were subclassified according to the simplified adenocarcinoma classification (Table 1), while 78 cases were excluded as not eligible for the study (10.3\%). From these excluded cases, $33.3 \%$ were classified as non-adenocarcinomas, $29.5 \%$ were adenocarcinomas of non-cervical origin, and the rest were not evaluated due to non-available material. Among the finally included cases, classic adenocarcinomas accounted for $83.1 \%$ of cases, while rare histological variants accounted for a few percent of cases individually (Table 2).

HPV positivity varied significantly between the different histologic tumor subtypes. Classic adenocarcinomas showed $71.8 \%$ HPV positivity (Table 3), while other adenocarcinoma tumor types showed low prevalence of HPV. HPV was detected in $27.3 \%$ of endometrioid adenocarcinoma, $25 \%$ of serous, $20 \%$ of clear cell, $13.9 \%$ of not otherwise specified, and $8.3 \%$ in minimal deviation adenocarcinomas. In all, $91.8 \%$ of HPV-positive tumors showed the presence of a single viral type and in $7 \%$ of cases multiple viral types were detected. Unknown HPV type was encountered in $1.2 \%$ of cases (Table 3 ).

The average age of the patients with classic adenocarcinoma was 50.5 years (Table 4). Patients with the diagnosis of serous and not otherwise specified were on average a decade older. The youngest patient (16 years) was recorded in the clear cell group. Patients with HPV-positive tumors were on average younger than patients with HPVnegative tumors; however, this difference was statistically significant only for classic adenocarcinomas and did not reach statistical significance in other tumor subtypes probably due to small number of cases.

We have analyzed HPV-negative classic adenocarcinoma cases in more detail (Table 5). When stratified by continent of origin, HPV negativity ranged from $1 / 4$ to $1 / 3$ of cases in all continents (from $25.1 \%$ in Asia to $32.8 \%$ in Africa, adjusted values), with exception of North American cases which showed $8.9 \%$ HPV negativity. However, there were only 10 cases in this group and therefore the difference did not reach statistical significance. The duration of tissue storage had a statistically significant impact on HPV detection ( $P$-trend value $=0.004)$. Cases from the last 30 years showed HPV negativity ranging from 22.2 to $26.8 \%$, however, older cases had significantly lower HPV detection rate, with HPV negativity exceeding 50\% in cases stored for $>60$ years. Age of the patients at the time of the diagnosis was another variable showing direct correlation with HPV negativity (Table 5), further corroborating age data presented in Table 4. Among patients aged 40-49 years only 
Table 2 Adenocarcinoma histological subtypes distribution using the simplified classification system

\begin{tabular}{|c|c|c|c|}
\hline Histologic adenocarcinoma subtypes & $\mathrm{N}$ & $\%$ & $\%$ Among adenocarcinomas \\
\hline Classic & 567 & 74.6 & 83.1 \\
\hline Not otherwise specified & 36 & 4.7 & 5.3 \\
\hline Clear cell & 30 & 3.9 & 4.4 \\
\hline Serous & 24 & 3.2 & 3.5 \\
\hline Minimal deviation & 12 & 1.6 & 1.8 \\
\hline Endometrioid & 11 & 1.4 & 1.6 \\
\hline Mixed: serous and clear cell & 2 & 0.3 & 0.3 \\
\hline Subtotal adenocarcinomas & 682 & 89.7 & 100 \\
\hline \multicolumn{4}{|l|}{ Excluded cases after pathological subtype review } \\
\hline Insufficient material for pathological review & 29 & 3.8 & \\
\hline Adenosquamous carcinoma & 20 & 2.6 & \\
\hline Adenocarcinoma with a doubtful cervical origin & 13 & 1.7 & \\
\hline Adenocarcinoma of not cervical origin & 10 & 1.3 & \\
\hline Other non-epithelial neoplasias & 6 & 0.8 & \\
\hline Total & 760 & 100 & \\
\hline
\end{tabular}

$\%$ : percent among all adenocarcinomas initially included; \% among adenocarcinomas: percent among confirmed cervical adenocarcinomas after the pathological subtypes review.

Table 3 HPV positivity by histologic adenocarcinoma subtypes

\begin{tabular}{|c|c|c|c|c|c|c|c|c|c|}
\hline Histologic adenocarcinoma subtypes & $\mathrm{N}$ & $N H P V+$ & $\%$ & $\begin{array}{l}\text { Single HPV } \\
\text { infection }\end{array}$ & $\%^{a}$ & $\begin{array}{c}\text { Multiple } H P V \\
\text { infection }\end{array}$ & $\%^{a}$ & $\begin{array}{c}H P V \\
\text { unknown }\end{array}$ & $\%^{a}$ \\
\hline Classic & 567 & 407 & 71.8 & 375 & 92.1 & 27 & 6.6 & 5 & 1.2 \\
\hline Not otherwise specified & 36 & 5 & 13.9 & 4 & 80.0 & 1 & 20.0 & 0 & 0.0 \\
\hline Clear cell & 30 & 6 & 20.0 & 5 & 83.3 & 1 & 16.7 & 0 & 0.0 \\
\hline Serous & 24 & 6 & 25.0 & 6 & 100 & 0 & 0.0 & 0 & 0.0 \\
\hline Minimal deviation & 12 & 1 & 8.3 & 1 & 100 & 0 & 0.0 & 0 & 0.0 \\
\hline Endometrioid & 11 & 3 & 27.3 & 2 & 66.7 & 1 & 33.3 & 0 & 0.0 \\
\hline Mixed: serous and clear cell & 2 & 0 & 0.0 & - & - & - & - & - & - \\
\hline Total & 682 & 428 & 62.8 & 393 & 91.8 & 30 & 7.0 & 5 & 1.2 \\
\hline
\end{tabular}

$N+$ : HPV-positive adenocarcinomas; \%: HPV positivity among HPV analyzed adenocarcinomas; \% ${ }^{a}$ : percent among HPV-positive cases; HPV unknown: DEIA +/LiPA 25 .

17.5\% tumors were HPV-negative; but the negativity increased to $31.7 \%$ among patients aged $50-59$ years and to $43.1 \%$ in patients over 60 years old $(P$-trend value $<0.001)$. A group of 9486 squamous cell carcinomas obtained from the same institutions was used for comparison. The overall detection of HPV in squamous cell carcinomas was higher than in adenocarcinoma, 87.0 vs $71.8 \%$, respectively. HPV negativity was higher in squamous cell carcinomas originating from Latin America and Africa, in cases stored for $40-50$ years, and in patients older than 60 years old (Table 5), paralleling the trend seen in adenocarcinomas.

Table 6 shows HPV genotype distribution in adenocarcinomas by histologic subtype. Three HPV genotypes, HPVs 16, 18, and 45, dominated in all adenocarcinomas and together accounted for $94.1 \%$ of HPV-positive tumors. HPV16 was the most common and was found in $50.9 \%$ of HPV-positive cases, followed by HPV18 (31.6\%) and HPV45 (11.6\%). Other HPV types including HPV31, 51, $59,33,35,6,30,39,53,68$, or 73 were found in lower proportions and except for one case HPV30 identified in not otherwise specified adenocarcinomas, all these other HPV types were detected in the classic histological subtype.

\section{Discussion}

The study represents the largest and the broadest analysis of HPV prevalence and type distribution in different subtypes of cervical adenocarcinoma to date. We were able to collect 682 tumor cases from 5 continents. The overall prevalence of HPV was $62.8 \%$ for all adenocarcinoma cases together; however, the prevalence showed variation depending on histologic tumor subtype, geographical region, patient age, and sample storage time. There were several prior studies examining HPV positivity in cervical adenocarcinomas in western countries including USA, ${ }^{4,5}$ South Korea, ${ }^{3,6}$ The Netherlands, ${ }^{7}$ and Italy, ${ }^{8}$ but the data from Eastern Europe, Africa, Latin America were scarce. The only prior 
Table 4 Age at diagnosis by final histologic adenocarcinoma subtypes and HPV positivity

\begin{tabular}{|c|c|c|c|c|c|c|c|c|c|c|c|c|c|c|c|}
\hline \multirow{2}{*}{$\begin{array}{l}\text { Histologic } \\
\text { adenocarcinoma } \\
\text { subtypes }\end{array}$} & \multicolumn{7}{|c|}{$A l l^{\mathrm{a}}$} & \multicolumn{4}{|c|}{ HPV-negative } & \multicolumn{4}{|c|}{$H P V$-positive } \\
\hline & $\mathrm{N}$ & Mean & s.d. & $95 \% C I$ & Median & Min-Max & $\mathrm{N}$ & Mean & s.d. & $95 \% C I$ & $\mathrm{~N}$ & Mean & s.d. & $95 \% C I$ & $\mathrm{P}$-value ${ }^{\$}$ \\
\hline Classic & 471 & 50.5 & 12.6 & $49.4-51.7$ & 48.0 & $24-90$ & 130 & 55.1 & 13.3 & $52.8-57.4$ & 341 & 48.8 & 11.9 & $47.5-50.1$ & $<0.001$ \\
\hline $\begin{array}{l}\text { Not otherwise } \\
\text { specified }\end{array}$ & 27 & 59.7 & 14.2 & $54.1-65.3$ & 63.0 & $32-82$ & 24 & 59.4 & 14.6 & $53.3-65.6$ & 3 & 62.0 & 13.0 & $29.7-94.3$ & 0.7718 \\
\hline Clear cell & 28 & 53.1 & 17.5 & $46.4-59.9$ & 56.0 & $16-84$ & 23 & 54.6 & 18.2 & $46.7-62.4$ & 5 & 46.6 & 13.6 & $29.7-63.5$ & 0.3646 \\
\hline Serous & 21 & 61.4 & 14.6 & $54.8-68.1$ & 64.0 & $25-86$ & 16 & 64.5 & 12.7 & $57.8-71.3$ & 5 & 51.6 & 17.3 & $30.1-73.1$ & 0.0838 \\
\hline Minimal deviation & 11 & 53.1 & 13.5 & $44.1-62.1$ & 53.0 & $34-76$ & 10 & 54.2 & 13.6 & $44.4-64.0$ & 1 & 42.0 & 0.0 & - & - \\
\hline Endometrioid & 10 & 44.9 & 11.3 & $36.8-53.0$ & 46.5 & $27-63$ & 7 & 47.7 & 11.9 & $36.7-58.8$ & 3 & 38.3 & 7.2 & $20.4-56.3$ & 0.2474 \\
\hline $\begin{array}{l}\text { Mixed: serous and } \\
\text { clear cell }\end{array}$ & 2 & 59.0 & 26.9 & $0.0-300.4$ & 59.0 & $40-78$ & 2 & 59.0 & 26.9 & $0.0-300.4$ & 0 & - & - & - & - \\
\hline Total & 570 & 51.5 & 13.3 & $50.4-52.6$ & 50.0 & $16-90$ & 212 & 56.0 & 14.2 & $54.0-57.9$ & 358 & 48.8 & 12.0 & $47.6-50.1$ & $<0.001$ \\
\hline
\end{tabular}

Abbreviations: $N$ : number of cases with age information; s.d.: standard deviation; 95\% CI: 95\% confidence interval; Min-Max: minimum and maximum values; $\$$ : $P$-value for the mean age comparison by HPV positivity.

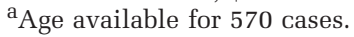

multicontinental study was conducted by IARC ${ }^{9}$ and consisted of cases from North Africa, South America, and Southeast Asia. The overall HPV prevalence in that study was reported as 93\% $(n=157$ cases); however, the study combined adenocarcinomas with adenosquamous carcinomas which accounted for one-third of all cases. The study of Castellsagué et al did not identify regional differences in overall HPV positivity. In two studies from South Korea, the overall HPV positivity was $88.5 \%(n=113$ cases $)$ and $90.3 \% \quad(n=196$ cases $)$, respectively. ${ }^{3,6}$ In a study from the Netherlands, the overall positivity was reported at $94 \% \quad(n=77$ cases). ${ }^{7}$ Several factors may be contributing to our lower rate of HPV detection. The aging of the paraffin-embedded tissue samples and differences in regional tissue fixation protocols with use of nonbuffered formalin may be significant factors for decreased HPV detection. We have observed a significantly lower HPV detection in cases stored over 30 years and in cases acquired from nonwestern countries, and similar trend but to a lesser degree was also seen in our reference group of squamous cell carcinomas. Prior study of HPV detection in adenocarcinomas has identified that use of formalin-fixed tissue may yield a lower HPV detection rate as compared with fresh-frozen tissue. ${ }^{10}$ HPV detection in adenocarcinomas poses a difficult technical problem due to the relatively low viral load in this type of malignancy. Glandular epithelium does not support productive HPV infection and therefore there is no accumulation of replicated episomal HPV DNA in the infected cells, and only a low copy number of HPV DNA is present integrated into the cell genome.,11 This is in contrast to squamous cell carcinomas, in which malignant squamous epithelium supports viral replication. As a result, a high copy number of replicated episomal HPV DNA along with integrated virus is present in the infected cells. These may be the reasons why, historically, the reported prevalence of HPV in squamous cell carcinomas was significantly higher than in adenocarcinoma. ${ }^{12}$ Aside from the technical aspects that may affect HPV detection in adenocarcinomas, there may be actual geographical variability of HPV positivity in adenocarcinomas. Future prospective studies with standardized tissue procurement will be able to address this issue in more detail.

In our study, we have performed a consensus histopathologic review of adenocarcinoma cases with subtyping according to a simplified scheme of WHO adenocarcinoma classification. HPV showed high prevalence in classic cervical adenocarcinoma $(71.8 \%)$ and much lower prevalence in rare histologic subtypes of adenocarcinoma with range from $27.3 \%$ in the endometrioid subtype to $8.3 \%$ in the minimal deviation cases. These results are consistent with findings from prior small series. Recent study of unusual variants of adenocarcinoma found no HPV DNA in a group of cases including clear cell $(n=9)$, gastric type $(n=11)$, minimal deviation $(n=3)$, mesonephric type $(n=1)$, except a single case of serous adenocarcinoma positive for HPV16. ${ }^{13}$ Houghton et al ${ }^{14}$ reported no HPV detection in four clear cell, three minimal deviation, and three mesonephric adenocarcinomas, and positive amplification of HPV16 in a single case of serous adenocarcinoma. In a study by Pirog et $a l,{ }^{4} \mathrm{HPV}$ DNA was not detected in any of four clear cell, two minimal deviation, one serous, and one mesonephric adenocarcinoma. The limitation of all these prior studies was low number of cases available for testing. Our current study is the largest to date report of HPV DNA detection in unusual adenocarcinoma variants and our results clearly indicate that pathogenesis of majority of these tumors is not related to HPV infection.

The pathogenesis of HPV-negative endocervical adenocarcinomas has not been yet elucidated. Minimal deviation and gastric type adenocarcinoma were shown to arise from a common precursor referred to as lobular endocervical glandular hyperplasia. ${ }^{15}$ Approximately $10 \%$ of minimal deviation 
Table 5 HPV negativity of classic adenocarcinoma $(N=567)$ and squamous cell carcinoma $(N=9486)$ by continent, time at diagnosis, and age at diagnosis

\begin{tabular}{|c|c|c|c|c|c|c|c|c|c|c|c|c|c|c|}
\hline \multirow[b]{3}{*}{ Characteristics } & \multicolumn{14}{|c|}{ HPV negativity } \\
\hline & \multicolumn{7}{|c|}{ Adenocarcinoma-classic type } & \multicolumn{7}{|c|}{ Squamous cell carcinoma } \\
\hline & $N$ & $N-$ & $\%$ & Adjusted $^{a} \mathrm{OR}$ & $95 \% C I$ & Adjusted ${ }^{a} \%$ & $95 \% C I$ & $N$ & $N-$ & $\%$ & Adjusted $^{a}$ OR & $95 \% C I$ & Adjusted $^{a} \%$ & $95 \% C I$ \\
\hline \multicolumn{15}{|l|}{ Region } \\
\hline Europe & 151 & 46 & 30.5 & 0.89 & $0.51-1.55$ & 28.9 & $22.0-37.0$ & 2093 & 203 & 9.7 & 0.92 & $0.75-1.13$ & 9.4 & $8.2-10.7$ \\
\hline North America & 10 & 1 & 10.0 & 0.24 & $0.03-2.08$ & 8.9 & $1.2-44.3$ & 160 & 12 & 7.5 & 0.78 & $0.42-1.42$ & 7.4 & $4.2-12.6$ \\
\hline Latin America & 206 & 58 & 28.2 & 0.80 & $0.47-1.34$ & 26.4 & $20.6-33.1$ & 3764 & 632 & 16.8 & 1.56 & $1.33-1.84$ & 16.3 & $15.1-17.5$ \\
\hline Africa & 42 & 14 & 33.3 & 1.38 & $0.62-3.06$ & 32.8 & $20.3-48.4$ & 609 & 118 & 19.4 & 2.53 & $1.96-3.26$ & 19.1 & $16.2-22.5$ \\
\hline Asia & 156 & 41 & 26.3 & Ref. & & 25.1 & $18.7-32.6$ & 2722 & 264 & 9.7 & Ref. & & 9.5 & $8.4-10.6$ \\
\hline Oceania & 2 & 0 & 0.0 & NA & - & NA & - & 138 & 5 & 3.6 & 0.37 & $0.15-0.92$ & 3.6 & 1.5-8.3 \\
\hline \multicolumn{15}{|l|}{ Time at diagnosis } \\
\hline $1940-49$ & 9 & 5 & 55.6 & 6.19 & $1.40-27.32$ & 55.6 & $25.1-82.3$ & 99 & 12 & 12.1 & 1.31 & $0.69-2.50$ & 11.8 & $6.8-19.8$ \\
\hline $1950-59$ & 26 & 10 & 38.5 & 2.98 & $1.13-7.82$ & 38.2 & $21.7-58.1$ & 483 & 65 & 13.5 & 1.24 & $0.91-1.69$ & 13.1 & $10.4-16.4$ \\
\hline 1960-69 & 41 & 10 & 24.4 & 1.37 & $0.59-3.19$ & 23.7 & $13.1-39.1$ & 909 & 197 & 21.7 & 2.31 & $1.86-2.88$ & 21.3 & $18.7-24.1$ \\
\hline $1970-79$ & 87 & 36 & 41.4 & 2.53 & $1.35-4.73$ & 41.0 & $30.9-51.9$ & 1171 & 215 & 18.4 & 1.71 & $1.39-2.10$ & 17.9 & $15.8-20.2$ \\
\hline 1980-89 & 61 & 17 & 27.9 & 1.41 & $0.69-2.88$ & 26.8 & $17.1-39.4$ & 1370 & 176 & 12.8 & 1.16 & $0.94-1.43$ & 12.4 & $10.7-14.2$ \\
\hline 1990-99 & 151 & 35 & 23.2 & Ref. $^{b}$ & & 22.2 & $16.2-29.8$ & 2451 & 257 & 10.5 & Ref. $^{b}$ & & 9.9 & $8.8-11.2$ \\
\hline 2000-09 & 192 & 47 & 24.5 & 1.19 & $0.70-2.03$ & 23.0 & $17.5-29.7$ & 3003 & 312 & 10.4 & 0.93 & $0.78-1.11$ & 9.9 & $8.9-11.1$ \\
\hline \multicolumn{15}{|c|}{ Age at diagnosis (years) } \\
\hline$<40$ & 97 & 19 & 19.6 & Ref. $^{b}$ & & 18.7 & $12.1-27.7$ & 1499 & 178 & 11.9 & Ref. $^{b}$ & & 11.0 & $9.5-12.6$ \\
\hline $40-49$ & 154 & 28 & 18.2 & 0.97 & $0.50-1.87$ & 17.5 & $12.3-24.4$ & 2267 & 282 & 12.4 & 1.09 & $0.89-1.34$ & 11.5 & $10.3-12.9$ \\
\hline $50-59$ & 103 & 33 & 32.0 & 2.12 & $1.09-4.13$ & 31.7 & $23.4-41.3$ & 2003 & 248 & 12.4 & 1.13 & $0.92-1.39$ & 11.6 & $10.2-13.0$ \\
\hline$>59$ & 117 & 50 & 42.7 & 3.55 & $1.86-6.78$ & 43.1 & $34.2-52.5$ & 2140 & 312 & 14.6 & 1.46 & $1.19-1.79$ & 13.7 & $12.3-15.2$ \\
\hline Missing & 96 & 30 & 31.3 & 1.30 & $0.63-2.67$ & 30.3 & $21.8-40.5$ & 1577 & 214 & 13.6 & 0.99 & $0.78-1.24$ & 12.8 & $11.3-14.6$ \\
\hline Total & 567 & 160 & 28.2 & & & 26.7 & $23.0-30.7$ & 9486 & 1234 & 13.0 & & & 12.1 & $11.5-12.8$ \\
\hline
\end{tabular}

Abbreviations: 95\% CI: 95\% confidence interval; N: number of HPV DNA analyzed cases; $N-$ : number of HPV DNA-negative cases; NA: not available; OR: odds ratio. Statistically significant OR is highlighted in bold.

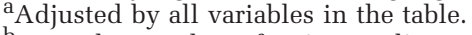

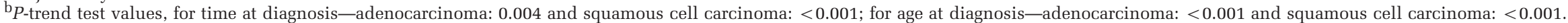


Table 6 Distribution of HPV genotypes in adenocarcinoma by histological subtype

\begin{tabular}{|c|c|c|c|c|c|c|c|c|c|c|c|c|c|c|c|c|c|c|c|c|}
\hline \multicolumn{3}{|c|}{$G L O B A L$} & \multicolumn{3}{|c|}{ Classic } & \multicolumn{3}{|c|}{ Not otherwise specified } & \multicolumn{3}{|c|}{ Clear cell } & \multicolumn{3}{|c|}{ Serous } & \multicolumn{3}{|c|}{ Minimal deviation } & \multicolumn{3}{|c|}{ Endometrioid } \\
\hline$H P V$ & $\mathrm{n}$ & $\%$ & $H P V$ & $\mathrm{n}$ & $\%$ & $H P V$ & $\mathrm{n}$ & $\%$ & $H P V$ & $\mathrm{n}$ & $\%$ & $H P V$ & $\mathrm{n}$ & $\%$ & $H P V$ & $\mathrm{n}$ & $\%$ & $H P V$ & $\mathrm{n}$ & $\%$ \\
\hline $\mathrm{HPV}+$ & 428 & 62.8 & $\mathrm{HPV}+$ & 407 & 71.8 & $\mathrm{HPV}+$ & 5 & 13.9 & $\mathrm{HPV}+$ & 6 & 20.0 & $\mathrm{HPV}+$ & 6 & 25.0 & $\mathrm{HPV}+$ & 1 & 8.3 & $\mathrm{HPV}+$ & 3 & 27.3 \\
\hline HPV 16 & 218 & 50.9 & HPV 16 & 202 & 49.7 & HPV 16 & 4 & 80.0 & HPV 16 & 4 & 66.7 & HPV 16 & 4 & 66.7 & HPV 16 & 1 & 100 & HPV 16 & 3 & 100 \\
\hline HPV 18 & 135 & 31.6 & HPV 18 & 130 & 32.1 & HPV 30 & 1 & 20.0 & HPV 18 & 2 & 33.3 & HPV 18 & 2 & 33.3 & & & & & & \\
\hline HPV 45 & 50 & 11.6 & HPV 45 & 50 & 12.3 & & & & & & & & & & & & & & & \\
\hline HPV 51 & 3 & 0.7 & HPV 51 & 3 & 0.8 & & & & & & & & & & & & & & & \\
\hline HPV 31 & 3 & 0.7 & HPV 59 & 3 & 0.7 & & & & & & & & & & & & & & & \\
\hline HPV 59 & 3 & 0.7 & HPV 31 & 3 & 0.7 & & & & & & & & & & & & & & & \\
\hline HPV 35 & 2 & 0.5 & HPV 35 & 2 & 0.5 & & & & & & & & & & & & & & & \\
\hline HPV 33 & 2 & 0.5 & HPV 33 & 2 & 0.5 & & & & & & & & & & & & & & & \\
\hline HPV 39 & 1 & 0.2 & HPV 53 & 1 & 0.2 & & & & & & & & & & & & & & & \\
\hline HPV 53 & 1 & 0.2 & HPV 6 & 1 & 0.2 & & & & & & & & & & & & & & & \\
\hline HPV 68 or 73 & 1 & 0.2 & HPV 68 or 73 & 1 & 0.2 & & & & & & & & & & & & & & & \\
\hline HPV 6 & 1 & 0.2 & HPV 39 & 1 & 0.2 & & & & & & & & & & & & & & & \\
\hline HPV 30 & 1 & 0.2 & HPV 68 & 1 & 0.2 & & & & & & & & & & & & & & & \\
\hline HPV 68 & 1 & 0.2 & & & & & & & & & & & & & & & & & & \\
\hline HPV U & 5 & 1.2 & HPVU & 5 & 1.2 & & & & & & & & & & & & & & & \\
\hline
\end{tabular}

Abbreviations: HPV + : HPV DNA positive; HPVU: HPV unknown (DEIA + / $\mathrm{LiPA}_{25}-$ ).

adenocarcinomas are associated with Peutz-Jeghers syndrome and mutation of the STK11/LKB1 gene. ${ }^{16}$ In the past, cervical clear cell carcinomas were linked to diethylstilbestrol exposure in utero; however, these tumors still rarely develop despite diethylstilbestrol discontinuation. Characteristically, this type of malignancy may present in very young subjects, as seen also in our study, suggesting possible developmental factors, paralleling diethylstilbestrol effects. ${ }^{17}$ Serous adenocarcinoma of the cervix in younger patients was described in familial ovarian cancer syndrome. ${ }^{18}$ Further, it has been described that over $90 \%$ of cervical serous cancers are positive for p53 immunostain, ${ }^{19}$ and therefore it is plausible that these tumors may develop similarly to endometrial serous carcinomas, as a result of p53 gene mutation in tubal-type epithelium.

Misclassification of tumor variants may confound the results of HPV testing in cervical adenocarcinoma. Precise tumor subclassification, such as differentiation of serous histological subtype from classic or minimal deviation from classic and clear cell, is often challenging due to overlapping morphologic features and results in, at best, moderate interobserver agreement. ${ }^{20}$ In our study, we established the final histologic diagnoses for the cases in a multistep consensus review. The difficulty was that for each case there was only one most representative tumor section available from the contributing institutions and the diagnosis had to be based on one slide selected by the referring pathologist. A follow-up study with immunoprofiling and laser-capture microdissection $^{21}$ of a subset of adenocarcinoma cases is being planned.

The finding of HPV negativity of unusual variants of endocervical adenocarcinoma has implications for the diagnosis and prevention of cervical adenocarcinoma, especially in the context of recent introduction of HPV testing and HPV vaccines. Fortunately, unusual variants of cervical adenocarcinomas are relatively rare. In the current study, they accounted for $17 \%$ of cases. Prior studies identified unusual adenocarcinoma subtypes in $11-16 \%$ of cases. ${ }^{4,13,22}$ HPV testing and vaccination will not cover most of these rare tumor variants, as well as some small proportion of classic adenocarcinomas. Cytologic screening is currently the only available screening method for these tumors. However, it is important to highlight that cytology poorly captures glandular lesions thus in addition to the low prevalence of these unusual types of adenocarcinomas, the diagnostic yield of cytologic screening is low.

Another observation in our study was that higher age at diagnosis of adenocarcinoma correlated with lower prevalence of HPV DNA. Similar trend was also seen in squamous cell carcinoma. The cause for this is uncertain. One may speculate that if the tumors in older patients had longer duration, HPV DNA may have been lost during tumor progression; alternatively, these tumors may have developed independently of HPV. The tumors in older patients may develop along different, non-HPV-dependent pathogenetic pathways, like it is seen for example in vulvar cancers.

HPV genotyping analysis carried out in our study showed a relatively narrow spectrum of HPV types present in adenocarcinoma cases. Three HPV genotypes, HPV 16, 18, and 45, accounted for $94.1 \%$ of HPV-positive cases. HPV types included in the vaccines (HPV16/18) were detected in $82.5 \%$ of HPV-positive cases. The ratio of HPV16 to HPV18 was 1.6. In the prior studies that examined the spectrum of HPV genotypes in adenocarcinomas, HPV16 and HPV18 together accounted for 75.7$94.8 \%$ of cases, with an average of $89.8 \%{ }^{4-7,9}$ While the percentage of combined HPV16 and 
HPV18-positive cases showed relative consistency between the studies, the ratio of HPV16 to HPV18 showed a significant variation. And thus some studies recorded marked HPV16 predominance ${ }^{5,9}$ while some reported HPV18 predominance. ${ }^{3,7,9}$ In a pooled analysis of 85 studies which included results on 1508 of combined adenocarcinomas and adenosquamous carcinomas ${ }^{12}$ HPV18 was found to be more common than HPV16 in Europe, Asia, and North America; however, this result is influenced by inclusion of adenosquamous carcinomas that are known for their association with HPV18. The relatively narrow spectrum of HPV genotypes detected in adenocarcinomas is very promising for the high efficacy of current prophylactic vaccination programs. The bivalent Cervarix HPV vaccine targeting HPV16 and HPV18 has been also shown to offer cross-protection against type HPV45, HPV31, and HPV33 leading to an estimated protection of $93.2 \% .{ }^{23}$ The quadrivalent Gardasil HPV vaccine does not appear to have a significant protective effect against type 45 but reduces infections with types HPV31, 33, 52, 39, 59, 35, and $58 .{ }^{24}$ In addition, a 9-valent HPV vaccine is currently under development and this vaccine will cover HPV16/18/31/33/45/52/58/6/11 offering a potential reduction close to $90 \%$ of invasive cervical cancer burden under a viral efficacy of $>95 \% .{ }^{25,26}$ Our results indicate that current and future HPV vaccines may prevent over $82 \%$ of HPV-positive adenocarcinoma cases.

Regarding potential HPV detection by the available HPV DNA screening tools, we have estimated the relative contribution and prevalence of HPV DNA targeted types by each of the FDA-approved tests (ie, Hybrid Capture 2, Cervista and Cobas 4800) in overall adenocarcinomas and in the different histological subtypes. The targeted HPV types are 13 high risk for Hybrid Capture 2 (HPVs 16, 18, 31, 33, $35,39,45,51,52,56,58,59$, and 68 ), and 14 for Cervista and Cobas 4800 (ie, adding HPV 66 to the previous list of 13 types). The detection rate of cases among HPV DNA-positive adenocarcinomas would be over $97 \%$, except for the not otherwise specified adenocarcinoma in which the detection would be of $80 \%$. If we take into account all the analyzed cases (prevalence), HPV screening would detect $61.3 \%$ of all the adenocarcinomas, $70 \%$ of the classic type, followed by the endometrioid subtype $(27.3 \%)$, serous $(25 \%)$, clear cell $(20 \%)$, not otherwise specified $(11.1 \%)$, and none of the two mixed cases (serous and clear cell).

Based on the results of the current study $37 \%$ of all adenocarcinomas and $28 \%$ of the classic subtypes are HPV DNA negative and with recent estimates that adenocarcinomas accounts for about $10 \%$ of cervical cancer globally we would estimate up to $3-4 \%$ of total cervical cancers to not contain HPV DNA and therefore could potentially be missed by HPV testing or not prevented by vaccination. The figures of $3-4 \%$ comply with the statement that virtually all cervical cancer cases are due to HPV infection ${ }^{27}$ and should not alter current views and preventive or clinical routine recommendations.

The limitation of our study is a use of a whole tissue section for HPV detection. The tumors were not microdissected before DNA extraction and therefore the results may be biased by detection of HPV present in the adjacent squamous epithelium or cervical mucus. As mentioned before a study with laser-capture microdissection and immunohistochemistry of the tumor tissue is being planned as a follow-up to the current study. The strengths of the study include multi-institutional consensus review of the cases and use of highly sensitive assays for HPV detection. Robust statistical methods were used to adjust the prevalence rates of the variables that were previously reported to affect the results. This detailed evaluation of the adenocarcinoma histological subtypes has allowed us to identify a group of rare subtypes largely unrelated to HPV. The classic adenocarcinoma showed a high HPV DNA detection rate. However, even in this group of tumors we still found a small proportion of HPVnegative cases. The results of our study may be of value for formulation of recommendations pertaining to second-generation polyvalent HPV vaccines. In addition, our findings underscore the importance of HPV types 16, 18, and 45 in type-specific highrisk HPV DNA-based screening tests.

\section{Acknowledgments}

We are grateful to the work of all the ICO team, DDL, the Steering Committee members, and to the participation of all the collaborating centers. The study is part of the international study RIS HPV TT coordinated at ICO, Barcelona, Spain. The study has been partially supported by Spanish public grants from the Instituto de Salud Carlos III (grants FIS PI030240, FIS PI061246, RCESP C03/09, RTICESP C03/10, RTIC RD06/0020/0095, RD12/0036/0056 and CIBERESP), from the Agència de Gestió d'Ajuts Universitaris i de Recerca (Catalan Government, grants AGAUR 2005SGR 00695 and 2009SGR126), the Marató de TV3 Foundation (051530), from Stichting Pathologie Ontwikkeling en Onderzoek (SPOO) foundation (The Netherlands), and the Lilly Foundation (Premio de Investigación Biomédica Preclínica 2012, F. Xavier Bosch). The field work was supported by unrestricted grants from GlaxoSmithKline Biologicals, Sanofi Pasteur MSD \& Merck \& Co, Inc., who had no role in the data collection, analysis, or interpretation of the results.

\section{Disclosure/conflict of interest}

DJ is a former scientific employee of GlaxoSmithKline and acts as a consultant on studies of HPV and cervical cancer to GlaxoSmithKline. FXB has 
received occasional lecture fees from GlaxoSmithKline, Merck, Sanofi Pasteur MSD, and Qiagen; and unrestricted grants through the Institution to conduct epidemiological and HPV vaccine studies from GlaxoSmithKline, Merck, Sanofi Pasteur MSD, Qiagen, and Roche. LA has received occasional travel fund to attend scientific meetings from Merck and Sanofi Pasteur MSD. SdS has received occasional travel fund to attend scientific meetings from Merck, Sanofi Pasteur MSD, and Qiagen; and unrestricted grants through the Institution to conduct epidemiological studies from GlaxoSmithKline and Merck.

\section{References}

1 de Sanjosé S, Quint WG, Alemany L, et al. Human papillomavirus genotype attribution in invasive cervical cancer: a retrospective cross-sectional worldwide study. Lancet Oncol 2010;11:1048-1056.

2 Wells M, Östör AG, Crum CP, et al. Chapter 5: Tumours of the uterine cervix, In: Tavassoli FA, Devilee P (eds). World Health Organization: Tumours of the Breast and Female Genital Organs (WHO/IARC Classification of Tumours) (IARC WHO Classification of Tumours). The International Agency for Research on Cancer: Lyon, France, 2003, pp 259-289.

3 Park JS, Kim YT, Lee A, et al. Prevalence and type distribution of human papillomavirus in cervical adenocarcinoma in Korean women. Gynecol Oncol 2013;130:115-120.

4 Pirog EC, Kleter B, Olgac S, et al. Prevalence of human papillomavirus DNA in different histological subtypes of cervical adenocarcinoma. Am J Pathol 2000;157: 1055-1062.

5 Quint KD, de Koning MN, Geraets DT, et al. Comprehensive analysis of Human Papillomavirus and Chlamydia trachomatis in in-situ and invasive cervical adenocarcinoma. Gynecol Oncol 2009;114:390-394.

6 An HJ, Kim KR, Kim IS, et al. Prevalence of human papillomavirus DNA in various histological subtypes of cervical adenocarcinoma: a population-based study. Mod Pathol 2005;18:528-534.

7 Zielinski GD, Snijders PJ, Rozendaal L, et al. The presence of high-risk HPV combined with specific p53 and p16INK4a expression patterns points to high-risk $\mathrm{HPV}$ as the main causative agent for adenocarcinoma in situ and adenocarcinoma of the cervix. J Pathol 2003;201:535-543.

8 Tenti P, Romagnoli S, Silini E, et al. Human papillomavirus types 16 and 18 infection in infiltrating adenocarcinoma of the cervix: PCR analysis of 138 cases and correlation with histologic type and grade. Am J Clin Pathol 1996;106:52-56.

9 Castellsagué X, Díaz M, de Sanjosé S, et al. International Agency for Research on Cancer Multicenter Cervical Cancer Study Group. Worldwide human papillomavirus etiology of cervical adenocarcinoma and its cofactors: implications for screening and prevention. J Natl Cancer Inst 2006;98:303-315.

10 Odida M, de Sanjosé S, Sandin S, et al. Comparison of human papillomavirus detection between freshly frozen tissue and paraffin embedded tissue of invasive cervical cancer. Infect Agent Cancer 2010;5:15.
11 Cooper K, Herrington CS, Lo ES, et al. Integration of human papillomavirus types 16 and 18 in cervical adenocarcinoma. J Clin Pathol 1992;45:382-384.

12 Clifford GM, Smith JS, Plummer M, et al. Human papillomavirus types in invasive cervical cancer worldwide: a meta-analysis. Br J Cancer 2003;88: 63-73.

13 Park KJ, Kiyokawa T, Soslow RA, et al. Unusual endocervical adenocarcinomas: an immunohistochemical analysis with molecular detection of human papillomavirus. Am J Surg Pathol 2011;35:633-646.

14 Houghton O, Jamison J, Wilson R, et al. p16 immunoreactivity in unusual types of cervical adenocarcinoma does not reflect human papillomavirus infection. Histopathology 2010;57:342-350.

15 Mikami Y, Kiyokawa T, Hata S, et al. Gastrointestinal immunophenotype in adenocarcinomas of the uterine cervix and related glandular lesions: a possible link between lobular endocervical glandular hyperplasia/ pyloric gland metaplasia and 'adenoma malignum'. Mod Pathol 2004;17:962-972.

16 Kuragaki C, Enomoto T, Ueno Y, et al. Mutations in the STK11 gene characterize minimal deviation adenocarcinoma of the uterine cervix. Lab Invest 2003;83: 35-45.

17 Ahrens WA, Barrón-Rodriguez LP, McKee M, et al. Clear cell adenocarcinoma of the cervix in a child without in utero exposure to diethylstilbestrol: a case report and review of the literature. Pediatr Dev Pathol 2005;8:690-695.

18 Kaplan EJ, Caputo TA, Shen PU, et al. Familial papillary serous carcinoma of the cervix, peritoneum, and ovary: a report of the first case. Gynecol Oncol 1998;70:289-294.

19 Nofech-Mozes S, Khalifa MM, Ismiil N, et al. Detection of HPV-DNA by a PCR-based method in formalin-fixed, paraffin-embedded tissue from rare endocervical carcinoma types. Appl Immunohistochem Mol Morphol 2010;18:80-85.

20 Alfsen GC, Reed W, Abeler VM. Reproducibility of classification in non-squamous cell carcinomas of the uterine cervix. Gynecol Oncol 2003;90:282-289.

21 Quint W, Jenkins D, Molijn A, et al. One virus, one lesion-individual components of CIN lesions contain a specific HPV type. J Pathol 2012;227:62-71.

22 Wang SS, Sherman ME, Silverberg SG, et al. Pathological characteristics of cervical adenocarcinoma in a multi-center US-based study. Gynecol Oncol 2006;103: $541-546$.

23 Lehtinen M, Dillner J. Clinical trials of human papillomavirus vaccines and beyond. J Nat Rev Clin Oncol 2013;10:400-410.

24 Schiller JT, Castellsagué X, Garland SM. A review of clinical trials of human papillomavirus prophylactic vaccines. Vaccine 2012;30:123-138.

25 Serrano B, Alemany L, Tous S, et al. Potential impact of a nine-valent vaccine in human papillomavirus related cervical disease. Infect Agent Cancer 2012; 7:38.

26 Luxembourg A. On behalf of the V503 Program Team Merck: Whitehouse Station, NJ, USA An overview of the 9-valent HPV L1 virus-like particle vaccine clinical development program. EUROGIN: Florence, 2013.

27 Walboomers JM, Jacobs MV, Manos MM, et al. Human papillomavirus is a necessary cause of invasive cervical cancer worldwide. J Pathol 1999;189: 12-19. 